\title{
Hadwiger and Helly-Type Theorems for Disjoint Unit Spheres in $\mathbb{R}^{3}$
}

\author{
Otfried Cheong* \\ Division of Computer Science \\ KAIST \\ Daejeon, South Korea \\ otfried@kaist.ac.kr
}

\author{
Xavier Goaoc ${ }^{\dagger}$ \\ Dept. of Mathematics and Computer Science \\ TU Eindhoven \\ Eindhoven, The Netherlands \\ goaoc@loria.fr
}

\author{
Andreas Holmsen \\ Department of Mathematics \\ University of Bergen \\ Bergen, Norway \\ andreash@mi.uib.no
}

\begin{abstract}
Let $\mathcal{S}$ be an ordered set of disjoint unit spheres in $\mathbb{R}^{3}$. We show that if every subset of at most six spheres from $\mathcal{S}$ admits a line transversal respecting the ordering, then the entire family has a line transversal. Without the order condition, we show that the existence of a line transversal for every subset of at most 11 spheres from $\mathcal{S}$ implies the existence of a line transversal for $\mathcal{S}$.
\end{abstract}

Categories and Subject Descriptors: F.2.2 [Nonnumerical Algorithms and Problems]: Geometrical problems and computations

\section{General Terms: Theory}

Keywords: Line transversal, unit sphere, unit ball, Hadwiger theorem, Helly theorem

\section{INTRODUCTION}

Helly's theorem [9] states that a finite family of convex sets in $\mathbb{R}^{d}$ has non-empty intersection if and only if any subfamily of size at most $d+1$ has non-empty intersection. Results of the type "if every subset of size $k$ of a set $\mathcal{S}$ has property $\mathcal{P}$ then $\mathcal{S}$ has property $\mathcal{P}$ " are therefore called Hellytype theorems and have been the object of active research in combinatorial geometry.

*Otfried Cheong was supported by the Brain Korea 21 Project, The School of Information Technology, KAIST, 2005.

†Xavier Goaoc was supported by the Netherlands' Organisation for Scientific Research (NWO) under project no. 639.023.301.

Permission to make digital or hard copies of all or part of this work for personal or classroom use is granted without fee provided that copies are not made or distributed for profit or commercial advantage and that copies bear this notice and the full citation on the first page. To copy otherwise, to republish, to post on servers or to redistribute to lists, requires prior specific permission and/or a fee.

SCG'05, June 6-8, 2005, Pisa, Italy.

Copyright 2005 ACM 1-58113-991-8/05/0006 ...\$5.00.
In 1958, Grünbaum [5] conjectured a Helly-type theorem for line transversals to a family $\mathcal{S}$ of pairwise disjoint translates of a compact convex figure in the plane (a line transversal for a set $\mathcal{S}$ of pairwise disjoint convex bodies in $\mathbb{R}^{d}$ is a line $\ell$ that intersects every element of $\mathcal{S}$ ): if every subfamily of $\mathcal{S}$ of size at most five admits a line transversal, then the entire family $\mathcal{S}$ has a line transversal. No Helly-type theorem holds for line transversals to planar convex sets in general: there are families $\mathcal{S}$ of $n$ convex figures with no line transversal such that every subset of $n-1$ figures has a line transversal. In 1957, however, Hadwiger [8] had shown that adding an ordering condition is sufficient to prove a similar statement: any ordered family $\mathcal{S}$ of compact convex figures in the plane such that every triple admits a line transversal compatible with the ordering of $\mathcal{S}$ admits a line transversal. (Surprising at first sight, the line transversal for $\mathcal{S}$ may not respect the ordering on $\mathcal{S}$, to prove the existence of a line transversal that respects the ordering on $\mathcal{S}$ one needs the assumption that any four-tuple admits an order-respecting line transversal.) Grünbaum's conjecture was finally proven by Tverberg [16] in 1989.

In three dimensions, neither Hadwiger nor Helly-type theorems exist for line transversals to convex objects, not even for translates of a convex compact set [12]. However, Hadwiger [7] proved a Helly-type theorem for line transversals to "thinly distributed" disjoint spheres in dimension $d$ with Helly number $d^{2}$. A family of spheres is thinly distributed if the distance between any two spheres is at least the sum of their radii. Grünbaum [6] improved this Helly number to $2 d-1$ using the topological Helly theorem. For the special case of congruent spheres - but without an additional assumption on their distribution-Holmsen et al. [11] showed a Hadwiger-type theorem with constant 12, and a Helly-type theorem with constant 46 . Their proof relies on a bound on the number of geometric permutations of congruent spheres by Katchalski et al. [13]. Using a more recent bound by Cheong et al. [2] the constants can be improved to 9 for the Hadwiger-type theorem and 18 for the Helly-type theorem.

For an overview of geometric transversal theory we refer to the surveys of Goodman et al. [4] and Wenger [17]. 
Interest for Helly-type theorems in the computational geometry community also stems from a connection existing between Helly-type theorems and LP-type problems, a class of optimization problems solvable by combinatorial linear programming approaches: Amenta [1] proved that an optimization problem is of LP-type if and only if the set of constraints that defines it admits a Helly-type theorem. Tight bounds on Helly numbers allow for more efficient resolution of the associated LP-type problems.

In this paper we give a new proof of a Hadwiger and a Helly-type theorem for line transversals to disjoint congruent spheres in $\mathbb{R}^{3}$. Our Hadwiger-type theorem is obtained in a way similar to the original proof by Hadwiger (for the planar case): we shrink the spheres uniformly until some subfamily has a single line transversal - in other words, the set of line transversals for the subfamily (with the correct order) consists of a single isolated element. We will say that the subfamily pins the line. The key step of the proof is an argument that shows that five spheres are always enough to pin a line, allowing for a Hadwiger-type theorem with constant six. We then derive a Helly-type theorem from the Hadwiger-type theorem by analyzing how the different geometric permutations may come into play. We refine the argument by Holmsen et al. [11] and establish that the Helly number is at most 11 .

Our proof that the "pinning number" is five is based on the topological Helly theorem [10]. The critical step is to show that in a suitable parametrization of line space, the set of line transversals to a given subfamily is a homology cell. Indeed, we show that the set of line transversals to a family of disjoint congruent spheres that induce the same ordering is contractible (in a suitable subset of $\mathbb{R}^{4}$ ), similar to Grünbaum's result on thinly distributed spheres [6]. The topological Helly theorem then immediately gives us a weak form of a Hadwiger-type theorem with constant five- a weak form because we need to restrict ourselves to a space of lines whose directions guarantee the right ordering on each subfamily (see Section 3 for details). Unfortunately, this does not seem to translate directly into Hadwiger number five for line transversals in general, but fortunately, we are able to use the "weak" Hadwiger-type theorem to prove that the pinning number is indeed five, which, as discussed above, then implies Hadwiger number six.

Still, the evidence of the "weak" Hadwiger-type theorem leads us to conjecture that the true Hadwiger number is probably five. (In fact, no lower bound better than four appears to be known.) For the Helly number, the discrepancy between the known lower bound of five and our upper bound of 11 is even larger - could it be that the true Helly number is in fact identical to the Hadwiger number?

\section{THE CONE OF DIRECTIONS}

A unit sphere is a sphere of radius one in $\mathbb{R}^{3}$. We consider spheres to be closed sets, so a tangent to a sphere intersects it and disjoint spheres are not allowed to touch. A line transversal for a set of disjoint unit spheres is an oriented line that intersects all the spheres. Let $\mathcal{S}$ be an ordered family of disjoint unit spheres, that is, we assume that a total order $\prec$ is defined on the family $\mathcal{S}$. A line transversal $\ell$ to a subset of $\mathcal{S}$ respects the order if whenever $\ell$ intersects a sphere $X \in \mathcal{S}$ before a sphere $Y \in \mathcal{S}$, we have $X \prec Y$.

Given a sphere $A$ and a direction $v$ in $\mathbb{R}^{3}$, we denote by $P_{v}(A)$ the two-dimensional disc obtained by projecting $A$ on a plane ${ }^{1}$ with normal $v$. Observe that a set of spheres $\mathcal{S}$ has a line transversal with direction $v$ if and only if the $\operatorname{discs}\left\{P_{v}(A) \mid A \in \mathcal{S}\right\}$ have a common intersection. We will abbreviate $\left\{P_{v}(A) \mid A \in \mathcal{S}\right\}$ as $P_{v}(\mathcal{S})$.

For an ordered family $\mathcal{S}$ of disjoint unit spheres, let $\mathcal{K}(\mathcal{S})$ be the set of unit vectors $v \in \mathbb{R}^{3}$ such that there is an order-respecting line transversal for $\mathcal{S}$ with direction vector $v$. Holmsen et al. [11] considered the case $|\mathcal{S}|=3$ and showed that $\mathcal{K}(\mathcal{S})$ is then a convex set (on the sphere of directions). This immediately generalizes to any $|\mathcal{S}|>3$, as a direct application of Helly's theorem for planar convex sets.

LEMma 1. Let $\mathcal{S}$ be an ordered family of disjoint unit spheres in $\mathbb{R}^{3}$. Then $\mathcal{K}(\mathcal{S})$ is a convex set.

Proof. The case $|\mathcal{S}| \leqslant 2$ is clear and $|\mathcal{S}|=3$ was proven by Holmsen et al. [11, Lemma 1]. For $|\mathcal{S}|>3$ we claim that $\mathcal{K}(\mathcal{S})=\bigcap_{\mathcal{S}^{\prime} \subset \mathcal{S},\left|\mathcal{S}^{\prime}\right|=3} \mathcal{K}\left(\mathcal{S}^{\prime}\right)$. One direction is obvious, so let $v \in \bigcap_{\mathcal{S}^{\prime} \subset \mathcal{S},\left|\mathcal{S}^{\prime}\right|=3} \mathcal{K}\left(\mathcal{S}^{\prime}\right)$. By choice of $v$, for any three spheres $A, B, C \in \mathcal{S}$ the $\operatorname{discs} P_{v}(\{A, B, C\})$ intersect. Thus, by Helly's theorem, the family $P_{v}(\mathcal{S})$ has non-empty intersection and the spheres of $\mathcal{S}$ have a common transversal with direction $v$. As this transversal respects the ordering induced by $\mathcal{S}$ on every triple of spheres, it respects the ordering on the whole family and thus $v \in \mathcal{K}(\mathcal{S})$. Therefore, $\mathcal{K}(\mathcal{S})$ is convex as the intersection of convex sets.

A line transversal is strict if it intersects each sphere without being tangent to it. For an ordered family $\mathcal{S}$ of disjoint unit spheres, let $\mathcal{K}^{\circ}(\mathcal{S})$ denote the set of unit vectors $v \in \mathbb{R}^{3}$ such that there is a strict order-respecting line transversal for $\mathcal{S}$ with direction vector $v$. Equivalently, $\mathcal{K}^{\circ}(\mathcal{S})$ is the set of unit vectors $v \in \mathbb{R}^{3}$ such that the intersection of the discs $P_{v}(\mathcal{S})$ has non empty interior. As expected, $\mathcal{K}^{\circ}(\mathcal{S})$ is the interior of $\mathcal{K}(\mathcal{S})$ when they are considered as subsets of the sphere of directions.

Lemma 2. Let $\mathcal{S}$ be an ordered family of disjoint unit spheres in $\mathbb{R}^{3}$. Then $\mathcal{K}^{\circ}(\mathcal{S})$ is the interior of $\mathcal{K}(\mathcal{S})$.

Proof. Given a sphere $A$, the $\operatorname{disc} P_{v}(A)$ depends continuously on $v$. Thus, for any $v_{0} \in \mathcal{K}^{\circ}(\mathcal{S})$ there exists a neighborhood of $v_{0}$ in which the intersection of the discs $P_{v}(\mathcal{S})$ has non-empty interior. By continuity, any line transversal to $\mathcal{S}$ with a direction in that neighborhood respects the ordering on $\mathcal{S}$, and so $\mathcal{K}^{\circ}(\mathcal{S}) \subset \operatorname{int}(\mathcal{K}(\mathcal{S}))$.
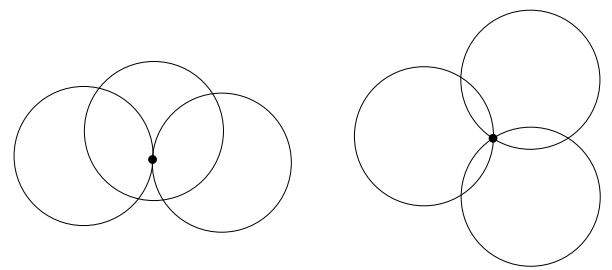

Figure 1. Possible configurations of three discs in the plane intersecting in a point.

Let now $v \in \operatorname{int}(\mathcal{K}(\mathcal{S})) \backslash \mathcal{K}^{\circ}(\mathcal{S})$. Since $v \notin \mathcal{K}^{\circ}(\mathcal{S})$ the intersection of the $\operatorname{discs} P_{v}(\mathcal{S})$ has empty interior. Hence, by Helly's theorem, there are three discs whose intersection

\footnotetext{
${ }^{1}$ The projections obtained using different such planes only differ by some translation.
} 

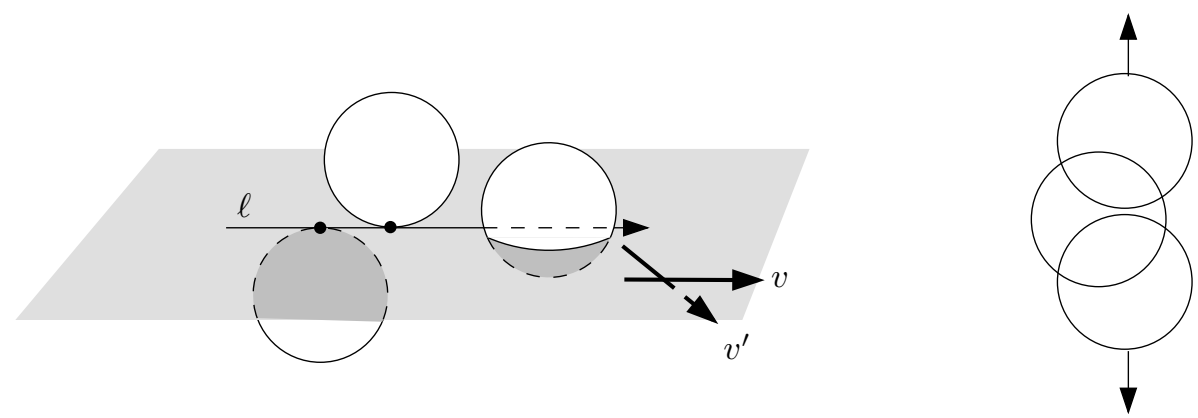

Figure 2. Perturbation removing all transversals when two discs are tangent: 3D view and projection on a plane with normal $v^{\prime}$.
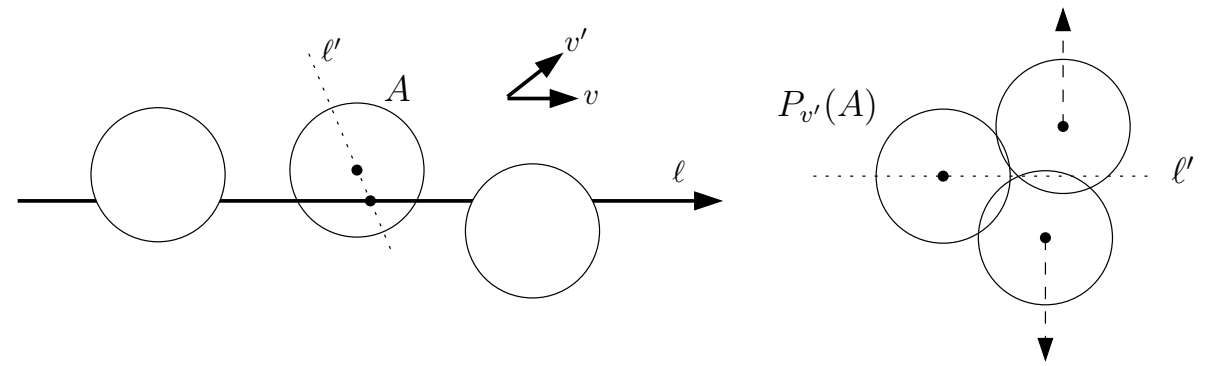

Figure 3. Perturbation removing all transversals when the intersection is on the boundary of the three discs.

has empty interior. The three discs must intersect since $v \in \mathcal{K}(\mathcal{S})$, so they intersect in a point and the three corresponding spheres have a unique transversal with direction $v$, denoted $\ell$. Figure 1 represents the two possible cases: either two of the discs are tangent or the common intersection point is on the boundary of all three discs. If two of the discs are tangent then $\ell$ lies in a plane separating the two corresponding spheres. There are then directions $v^{\prime}$ arbitrarily close to $v$ such that no transversal with direction $v^{\prime}$ for these two spheres exists (see Figure 2), a contradiction to $v \in \operatorname{int}(\mathcal{K}(\mathcal{S}))$. If the common intersection point is on the boundary of all three discs then $\ell$ is tangent to the three corresponding spheres. Let $A$ be the middle sphere (with respect to the ordering) and let $\ell^{\prime}$ be the line through the center of $A$ and its contact point with $\ell$ (see Figure 3 ). Consider a rotation of $v$ with a small angle $\delta$ around $\ell^{\prime}$. This rotation keeps $P_{v}(A)$ fixed, and moves the centers of the two other discs along lines orthogonal to $\ell^{\prime}$, either both away from $\ell^{\prime}$ or both towards $\ell^{\prime}$, depending on the sign of $\delta$. Any sufficiently small rotation that moves the centers away from $\ell^{\prime}$ turns $v$ into a direction $v^{\prime}$ such that no transversal for the three spheres exists in the direction $v^{\prime}$. Again, this contradicts the assumption that $v \in \operatorname{int}(\mathcal{K}(\mathcal{S}))$. It follows that $\operatorname{int}(\mathcal{K}(\mathcal{S})) \backslash \mathcal{K}^{\circ}(\mathcal{S})=\emptyset$, which concludes the proof.

Similar arguments prove the following lemma.

Lemma 3. Let $\mathcal{S}$ be an ordered family of disjoint unit spheres in $\mathbb{R}^{3}$. Then $\mathcal{K}(\mathcal{S})$ is either empty, a point, or has non-empty interior.

Proof. Assume that $\mathcal{K}(\mathcal{S})$ has empty interior and let $v \in \mathcal{K}(\mathcal{S})$. By Lemma $2, \mathcal{K}^{\circ}(\mathcal{S})$ is empty and the discs $P_{v}(\mathcal{S})$ have a common intersection with empty interior: a single point, $p(v)$, that lies on the boundary of $k \geqslant 2$ discs.
If $k=2$ then a perturbation similar to the first perturbation used in the proof of Lemma 2 yields a direction $v^{\prime}$ arbitrarily close to $v$ such that $v^{\prime} \in \mathcal{K}^{\circ}(\mathcal{S})$, contradicting the assumption that $\mathcal{K}(\mathcal{S})$ has empty interior. If $k=3$, an argument similar to the second perturbation used in the proof of Lemma 2 gives the same conclusion provided that the three discs are distinct. If they are not distinct, then the corresponding spheres have a common tangent plane, which separates two of the spheres from the third (see Figure 4). If this separated sphere is the middle one, then the three spheres have a single order-respecting line transversal, and $\mathcal{K}(\mathcal{S})$ is a single point. Otherwise the line can be perturbed into a strict transversal to all three spheres, again contradicting the assumption that $\mathcal{K}(\mathcal{S})$ has empty interior.

It follows that for any $v \in \mathcal{K}(\mathcal{S}), p(v)$ lies on the boundary of at least four discs, and $v$ is therefore the direction of a common tangent to four spheres in $\mathcal{S}$. Macdonald et al. [14] proved that four unit spheres in $\mathbb{R}^{3}$ admit at most 12 directions of common tangent lines, thus $\mathcal{K}(\mathcal{S})$ is finite. As $\mathcal{K}(\mathcal{S})$ is convex by Lemma 1 , it consists of a single point.

\section{APPLYING THE TOPOLOGICAL HELLY THEOREM}

A generalization of Helly's theorem based on topology instead of convexity was originally given by Helly himself [10]. We will use a version proven by Debrunner using modern tools (singular homology) [3], as it allows us to work with open sets.

Theorem 1 (Topological Helly Theorem [3]). Let $\left\{X_{j}\right\}_{j \in J}$ be a finite family of open subsets of Euclidean dspace $\mathbb{R}^{d}$ such that the intersection $X_{j_{1}} \cap \cdots \cap X_{j_{r}}$ of each $r$ 

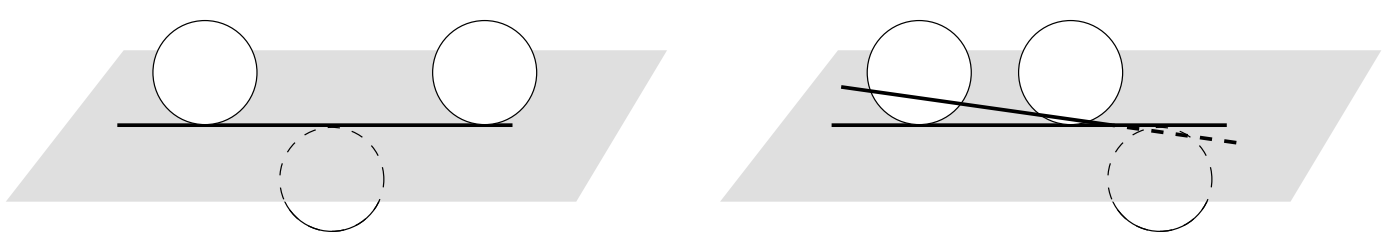

Figure 4. Three spheres with a common tangent plane.

sets of this family is nonempty for $r \leqslant d+1$ and is even a homology cell for $r \leqslant d$. Then $\bigcap_{j \in J} X_{j}$ is a homology cell.

Our aim is to apply Helly's topological theorem to sets of line transversals to disjoint unit spheres. Unfortunately, such a set is not necessarily a homology cell, and in fact not even connected: two lines intersecting disjoint objects in different orders cannot be in the same connected component of transversals to these objects. We overcome this difficulty by restricting the set of directions that we allow for transversals.

Let $D$ be a set of directions completely contained in the interior of a hemisphere, and let $\mathcal{L}(D)$ be the set of lines with directions in $D$. We parameterize $\mathcal{L}(D)$ as a subset of $\mathbb{R}^{4}$, using the points of intersection of a line $\ell \in \mathcal{L}(D)$ with two parallel planes that are not parallel to any direction in $D$.

For an ordered family $\mathcal{S}$ of disjoint unit spheres, let

$$
U(\mathcal{S}):=\{c(Y)-c(X) \mid X, Y \in \mathcal{S} ; X \prec Y\},
$$

where $c(X)$ denotes the center of sphere $X$. Let $D_{\mathcal{S}}$ be the set of directions making a positive dot-product with each $u \in U(\mathcal{S})$. Note that $D_{\mathcal{S}}$ is an open convex set on the sphere of directions. Clearly a line transversal $\ell \in \mathcal{L}\left(D_{\mathcal{S}}\right)$ for a subset $\mathcal{S}^{\prime} \subset \mathcal{S}$ respects the order on $\mathcal{S}^{\prime}$.

Let's now review a few notions from topology (these can be found, for instance, in the introductory chapter of Matoušek's book [15]). Given a topological space $A$ and a subset $B \subset A, B$ is a deformation retract of $A$ if there exists a continuous map $F: A \times[0,1] \rightarrow A$ such that

$$
\left\{\begin{array}{l}
F(a, 0)=a \text { for any } a \in A \\
F(b, t)=b \text { for any } b \in B \text { and } t \in[0,1] \\
F(a, 1) \in B \text { for any } a \in A
\end{array}\right.
$$

Two topological spaces $A, B$ are homotopy equivalent if there exists a third space $C$ such that both $A$ and $B$ are deformation retracts of $C$. A space that is homotopy equivalent to a single point is said to be contractible. Homology is invariant under homotopy equivalence, this implies in particular that a contractible space is a homology cell.

LEMma 4. Let $\mathcal{S}$ be an ordered family of at least two disjoint unit spheres in $\mathbb{R}^{3}$, and let $\mathcal{S}^{\prime} \subseteq \mathcal{S}$. Let $L$ be the set of lines in $\mathcal{L}\left(D_{\mathcal{S}}\right)$ that intersect the interior of all spheres in $\mathcal{S}^{\prime}$. Then $L$ is contractible.

Note the restriction on the direction of lines in $L$ : there may be strict order-respecting line transversals to $\mathcal{S}^{\prime}$ that are not in $L$.

Proof. Given a line $\ell \in L$, let $v_{\ell}$ be its direction. A transversal $\ell$ to $\mathcal{S}^{\prime}$ is barycentric if it goes through the center of mass of the intersection of $P_{v_{\ell}}\left(\mathcal{S}^{\prime}\right)$. For any direction $v$ in
$\mathcal{K}\left(\mathcal{S}^{\prime}\right)$ there is a unique barycentric transversal to $\mathcal{S}^{\prime}$, which we denote $b_{\mathcal{S}^{\prime}}(v)$.

Let $L^{*}$ denote the set of barycentric transversals to $\mathcal{S}^{\prime}$ with directions in $D_{\mathcal{S}}$. The projection of a sphere changes continuously with the direction of projection, so $b_{\mathcal{S}^{\prime}}$ is continuous. Since the direction of a line changes continuously with the line, $b_{\mathcal{S}^{\prime}}^{-1}$ is also continuous. Thus, $b_{\mathcal{S}^{\prime}}$ defines a homeomorphism between $L^{*}$ and $\mathcal{K}\left(\mathcal{S}^{\prime}\right) \cap D_{\mathcal{S}}$.

By Lemma $1, \mathcal{K}\left(\mathcal{S}^{\prime}\right)$ is convex and so is $D_{\mathcal{S}}$. Thus, $\mathcal{K}\left(\mathcal{S}^{\prime}\right) \cap$ $D_{\mathcal{S}}$ is convex and hence contractible. It follows that $L^{*}$ is also contractible. The map

$$
\left\{\begin{array}{l}
L \times[0,1] \rightarrow L \\
(\ell, t) \mapsto \ell+t\left(b_{\mathcal{S}^{\prime}}\left(v_{\ell}\right)-\ell\right)
\end{array}\right.
$$

is continuous and shows that $L^{*}$ is a deformation retract of $L$. Since $L^{*}$ is contractible, so is $L$.

We can now apply the topological Helly theorem to obtain a "weak" Hadwiger-type result.

LEMMA 5. Let $\mathcal{S}$ be an ordered family of at least five disjoint unit spheres in $\mathbb{R}^{3}$. If every subfamily $\mathcal{S}^{\prime} \subset \mathcal{S}$ of five spheres admits a strict line transversal with a direction in $D_{\mathcal{S}}$, then $\mathcal{S}$ admits a line transversal.

Proof. We apply Theorem 1 on $\mathcal{L}\left(D_{\mathcal{S}}\right)$. With the parameterization discussed above, $\mathcal{L}\left(D_{\mathcal{S}}\right) \subset \mathbb{R}^{4}$. For $S \in \mathcal{S}$ let $X_{S}$ be the subset of $\mathcal{L}\left(D_{\mathcal{S}}\right)$ of lines intersecting the interior of sphere $S$. Clearly, $X_{S}$ is an open set in $\mathbb{R}^{4}$. Consider now the intersection $Y:=X_{S_{1}} \cap \cdots \cap X_{S_{r}}$ of $r$ such sets. The set $Y$ consists of exactly those lines in $\mathcal{L}\left(D_{\mathcal{S}}\right)$ that are strict transversals of $S_{1}, \ldots, S_{r}$. The assumption of the lemma implies that $Y \neq \emptyset$ for $r \leqslant 5$. By Lemma $4 Y$ is then contractible, and hence a homology cell. Theorem 1 now implies that $\bigcap_{S \in \mathcal{S}} X_{S} \neq \emptyset$, and so there is an order-respecting strict line transversal for $\mathcal{S}$.

In principle, Lemma 5 is the Hadwiger-type result we are looking for. Its drawback is that it requires a subfamily of spheres to have not only an order-respecting transversal, but one that, in a sense, respects the order on the entire family of spheres. In the next section we will work around this problem, at the expense of raising the Hadwiger constant from five to six.

\section{A HADWIGER-TYPE THEOREM}

We will use the standard shrinking process also used by Hadwiger [8]: fixing the centers, we simultaneously decrease the radius of all spheres, keeping them congruent, until some sixtuple of spheres is about to lose its order-respecting transversal. For an ordered family $\mathcal{S}$ of disjoint unit spheres and a real number $\varepsilon>0$, let $\mathcal{S}_{\varepsilon}$ denote the spheres of $\mathcal{S}$ shrunk 
to have radius $1-\varepsilon$. Our first observation is that, in the shrinking process, the set of order-respecting transversals to some spheres disappears by first reducing to a single line.

Lemma 6. Let $\mathcal{S}$ be an ordered family of disjoint unit spheres in $\mathbb{R}^{3}$ admitting an order-respecting line transversal $\ell$. If $\mathcal{S}_{\varepsilon}$ has no order-respecting line transversal for any $\varepsilon>0$, then $\ell$ is the only order-respecting line transversal of $\mathcal{S}$.

Proof. First, observe that $\mathcal{K}^{\circ}(\mathcal{S})$ is empty, as otherwise there would be an $\varepsilon>0$ such that $\mathcal{S}_{\varepsilon}$ has an orderrespecting transversal. Thus, $\mathcal{K}(\mathcal{S})$ is non-empty but has an empty interior and, by Lemma 3 , is reduced to a point $v$. Since $v \notin \mathcal{K}^{\circ}(\mathcal{S})$, the $\operatorname{discs} P_{v}(\mathcal{S})$ intersect in a unique point and it follows that $\ell$ is the only order-respecting transversal to $\mathcal{S}$.

An isolated line transversal for a family of objects $\mathcal{F}$ is a line transversal $\ell$ that is disconnected (in the space of oriented lines) from any other line transversal of $\mathcal{F}$. (For a more formal definition, $\ell$ is isolated if there is a $\delta>0$ such that $\mathcal{F}$ admits no line transversal $\ell^{\prime} \neq \ell$ such that the shortest distance between $\ell$ and $\ell^{\prime}$ and the angle between the direction vectors of $\ell$ and $\ell^{\prime}$ are both less than $\delta$.) We will say that an ordered family of objects $\mathcal{F}$ has pinning number $k$ if the following holds: if $\mathcal{S} \subset \mathcal{F}$ has an isolated line transversal $\ell$ then there exists $\mathcal{S}^{\prime} \subset \mathcal{S}$, with $\left|\mathcal{S}^{\prime}\right| \leqslant k$, such that $\ell$ is an isolated line transversal of $\mathcal{S}^{\prime}$. Hadwiger [8] uses the fact that the pinning number of planar convex sets is three. Our next lemma investigates the pinning number of disjoint unit spheres in $\mathbb{R}^{3}$ :

LEMMA 7. The pinning number of disjoint unit spheres in $\mathbb{R}^{3}$ is at most five.

Proof. Let $\mathcal{S}$ be a family of at least six disjoint unit spheres in $\mathbb{R}^{3}$ admitting an isolated line transversal $\ell$. Let $\prec$ be the order on $\mathcal{S}$ induced by $\ell$. Lemma 4 implies that the set of line transversals to $\mathcal{S}$ respecting $\prec$ is connected, and so $\ell$ is the only order-respecting line transversal of $\mathcal{S}$. Since $\ell$ is not a strict transversal, $\mathcal{S}$ has no strict order-respecting transversal. By Lemma 5 , there is a subfamily $\mathcal{S}^{\prime} \subset \mathcal{S}$ of five spheres that has no strict order-respecting transversal with direction in $D_{\mathcal{S}}$, that is $\mathcal{K}^{\circ}\left(\mathcal{S}^{\prime}\right) \cap D_{\mathcal{S}}=\emptyset$. However, $\mathcal{K}\left(\mathcal{S}^{\prime}\right) \cap D_{\mathcal{S}} \neq \emptyset$ since it contains the direction of $\ell$. Since $\mathcal{K}\left(\mathcal{S}^{\prime}\right)$ is convex, by Lemma 1 , and $D_{\mathcal{S}}$ is open, it follows that $\mathcal{K}^{\circ}\left(\mathcal{S}^{\prime}\right)=\emptyset$ and $\mathcal{S}^{\prime}$ has no strict order-respecting transversal at all. Now, $\mathcal{K}\left(\mathcal{S}^{\prime}\right)$ is non-empty but has empty interior so Lemma 3 yields that $\mathcal{K}\left(\mathcal{S}^{\prime}\right)$ is a single direction $v$. Since $v \notin \mathcal{K}^{\circ}\left(\mathcal{S}^{\prime}\right)$, the $\operatorname{discs} P_{v}\left(\mathcal{S}^{\prime}\right)$ intersect in a unique point and $\ell$ is the only order-respecting line transversal of $\mathcal{S}^{\prime}$, and is thus isolated.

We can now prove our main theorem.

THEOREM 2. Let $\mathcal{S}$ be an ordered family of at least six disjoint unit spheres in $\mathbb{R}^{3}$. If every subfamily $\mathcal{S}^{\prime} \subset \mathcal{S}$ of six spheres admits an order-respecting line transversal, then $\mathcal{S}$ admits a line transversal. If every subfamily $\mathcal{S}^{\prime} \subset \mathcal{S}$ of seven spheres admits an order-respecting line transversal, then $\mathcal{S}$ admits an order-respecting line transversal.

Note that in the first conclusion of the theorem the transversal to $\mathcal{S}$ is not guaranteed to respect the order.
Proof. We simultaneously shrink all the spheres as long as every subfamily of size six has an order-respecting transversal. At some point, by Lemma 6 , a subfamily $\mathcal{S}^{\prime}$ of size six has a unique order-respecting transversal $\ell$. By Lemma 7 , there is then a subfamily $\mathcal{S}^{\prime \prime} \subset \mathcal{S}^{\prime}$ of at most five spheres, such that $\ell$ is the unique order-respecting transversal of $\mathcal{S}^{\prime \prime}$. For any sphere $X \in \mathcal{S} \backslash \mathcal{S}^{\prime \prime}$, the set $\mathcal{S}^{\prime \prime} \cup\{X\}$ has an order-respecting line transversal $\ell_{X}$. Since the only orderrespecting line transversal of $\mathcal{S}^{\prime \prime}$ is $\ell$, we must have $\ell_{X}=\ell$, and $\ell$ intersects $X$. It follows that $\ell$ is a line transversal for $\mathcal{S}$.

Similarly, if any subfamily of size seven admits an orderrespecting line transversal there exists a subfamily $\mathcal{S}^{\prime}$ of five spheres having a unique order-respecting line transversal $\ell$. For any $X, Y \in \mathcal{S}$ with $X \prec Y$, the subfamily $\mathcal{S}^{\prime} \cup\{X, Y\}$ admits an order-respecting line transversal that must be $\ell$, and $\ell$ intersects $X$ before $Y$. It follows that $\ell$ is an orderrespecting line transversal of $\mathcal{S}$.

\section{A HELLY-TYPE THEOREM}

We use the following result by Cheong et al. [2]

THEOREM 3 ([2]). Let $\mathcal{S}$ be a family of at least nine disjoint unit spheres in $\mathbb{R}^{d}$. Then $\mathcal{S}$ admits at most two distinct geometric permutations, which differ only in the swapping of two adjacent spheres.

This allows us to turn our Hadwiger-type theorem into a Helly-type theorem.

TheOREM 4. Let $\mathcal{S}$ be a family of at least 11 disjoint unit spheres in $\mathbb{R}^{3}$. If every subfamily $\mathcal{S}^{\prime} \subset \mathcal{S}$ of 11 spheres admits a line transversal, then $\mathcal{S}$ admits a line transversal.

Proof. We first shrink the spheres simultaneously until some subfamily $\mathcal{S}_{11}$ of 11 spheres is about to lose its last transversal (regardless of the ordering it induces).

If $\mathcal{S}_{11}$ admits more than one line transversal (all of which vanish if the spheres are shrunk any further), each transversal must realize a different geometric permutation. Theorem 3 then implies that $\mathcal{S}_{11}$ has exactly two line transversals, $\ell_{1}$ and $\ell_{2}$, with two distinct geometric permutations. By Lemma 7 , for each $\ell_{i}$ there are five spheres in $\mathcal{S}_{11}$ for which $\ell_{i}$ is the only line transversal respecting the ordering induced by $\ell_{i}$. There is thus a subfamily $\mathcal{S}^{\prime}$ of $\mathcal{S}_{11}$ of exactly 10 spheres (we can complete $\mathcal{S}^{\prime}$ using spheres from $\mathcal{S}_{11}$ if needed) for which $\ell_{1}$ and $\ell_{2}$ are the only line transversals respecting their respective orders. By Theorem $3 \mathcal{S}^{\prime}$ admits at most two geometric permutations, and so $\ell_{1}$ and $\ell_{2}$ are its only line transversals. Since any subfamily of 11 spheres has a line transversal, any sphere of $\mathcal{S} \backslash \mathcal{S}^{\prime}$ must intersect $\ell_{1}$ or $\ell_{2}$. If all the spheres intersect both lines then the theorem is proven. Otherwise, there exists a sphere $A$ that intersects, say, $\ell_{1}$ but not $\ell_{2}$. Then $\mathcal{S}^{\prime} \cup\{A\}$ is a family of 11 spheres with a unique transversal. We are left with a set $\mathcal{S}_{11}$ of 11 spheres that has a unique transversal $\ell$.

Let $\prec_{\ell}$ be the order on $\mathcal{S}_{11}$ induced by $\ell$. By Lemma 7 , there is a subfamily $\mathcal{S}_{5} \subset \mathcal{S}_{11}$ such that $\ell$ is the unique transversal of $\mathcal{S}_{5}$ respecting $\prec_{\ell}$. For each $Z \in \mathcal{S}_{11} \backslash \mathcal{S}_{5}$, let $\mathcal{S}_{Z}$ denote the set $\mathcal{S}_{11} \backslash\{Z\}$. If one of the subsets $\mathcal{S}_{Z}$ has no other transversal than $\ell$ then every other sphere of $\mathcal{S}$ intersects $\ell$ and the proof is complete. 
We now assume that every $\mathcal{S}_{Z}$ has some transversal $\ell_{Z}$ distinct from $\ell$ and obtain a contradiction. Since $\mathcal{S}_{Z}$ contains $\mathcal{S}_{5}, \ell_{Z}$ realizes a geometric permutation different from that of $\ell$. By Theorem 3, the order induced by $\ell_{Z}$ on $\mathcal{S}_{11}$ differs from $\prec_{\ell}$ by the swapping of two adjacent spheres $X, Y$. Since $\ell_{Z}$ realizes a geometric permutation of $\mathcal{S}_{5}$ different from $\ell$, we must have $X, Y \in \mathcal{S}_{5}$. Let $Z_{1}, Z_{2} \in \mathcal{S}_{11} \backslash \mathcal{S}_{5}$, and consider the set $\mathcal{S}_{11} \backslash\left\{Z_{1}, Z_{2}\right\}$. It admits the transversals $\ell$, $\ell_{Z_{1}}$, and $\ell_{Z_{2}}$ but, by Theorem 3 , at most two geometric permutations. Since $\ell$ is the unique transversal respecting $\prec_{\ell}$, $\ell_{Z_{1}}$ and $\ell_{Z_{2}}$ must realize the same geometric permutation on $\mathcal{S}_{11} \backslash\left\{Z_{1}, Z_{2}\right\}$. Thus the spheres $X, Y \in \mathcal{S}$ do not depend on the choice of $Z$. Let $\prec$ be the order on $\mathcal{S}_{11}$ obtained from $\prec_{\ell}$ by swapping $X$ and $Y$. For any $Z \in \mathcal{S}_{11} \backslash \mathcal{S}_{5}$ the subfamily $\mathcal{S}_{Z}$ admits a line transversal respecting $\prec$. On the other hand, $\mathcal{S}_{11}$ does not admit such a transversal as $\ell$ is its only transversal. By (the second half of) Theorem 2, there is a subset $\mathcal{S}_{7} \subset \mathcal{S}_{11}$ of at most seven spheres that does not admit a transversal respecting $\prec$. We must have $X, Y \in \mathcal{S}_{7}$, as without both $X$ and $Y, \prec_{\ell}$ and $\prec$ are equivalent. This implies that $\left|\mathcal{S}_{5} \cup \mathcal{S}_{7}\right| \leqslant 10$. There is therefore a $Z \in \mathcal{S}_{11} \backslash \mathcal{S}_{5}$ such that $\mathcal{S}_{5} \cup \mathcal{S}_{7} \subseteq \mathcal{S}_{Z}$. However, $\ell_{Z}$ cannot be a line transversal to $\mathcal{S}_{7}$, a contradiction.

\section{CONCLUSIONS}

If one can generalize Lemmas 1, 2 and 3 to dimensions higher than three, the remaining arguments will go through. This would imply that for line transversals to disjoint congruent spheres in $\mathbb{R}^{d}$, the pinning number is at most $2 d-1$, the Hadwiger number therefore at most $2 d$, and the Helly number at most $4 d-1$. The bottleneck here appears to be Lemma 1, the generalization of which we are currently studying.

Even in $\mathbb{R}^{3}$, it remains to close the gap between our upper bounds and the known lower bounds. As mentioned in the introduction, we are not aware of a lower bound for the Hadwiger number larger than four. Lemma 5 leads us to conjecture that the true Hadwiger number is five. To prove such a bound, one could attempt to understand in much more detail exactly how five spheres can pin a line transversal - our Lemma 7 doesn't provide much help there.

As for the Helly number, an example with spheres centered on the vertices of a regular pentagon shows that the Helly number is at least five. It would be interesting to at least know whether the Hadwiger and the Helly number are identical.

We proved a bound on the pinning number for unit spheres only. It would be interesting to see how far this can be generalized: perhaps smooth convex objects with positive curvature everywhere behave locally sufficiently like spheres to prove the same bound. For general convex objects - even smooth ones - the pinning number is at least six, as the following example using six unit-radius cylinders in $\mathbb{R}^{3}$ by Günter Rote shows: the first three cylinders are parallel to the $x$-axis, and their axes go through the points $(0,1,0)$, $(0,-1,1)$, and $(0,1,2)$, respectively. The remaining three cylinders are parallel to the $y$-axis, and their axes go through the points $(1,0,10),(-1,0,11)$, and $(1,0,12)$, respectively. The $z$-axis is the only transversal for all six cylinders, but any five of them have an infinite number of transversals.

\section{Acknowledgments}

We thank Gregory Ginot for helpful discussions and suggesting the proof of Lemma 4, and Günter Rote for the lower bound construction for the pinning number of cylinders.

\section{References}

[1] N. Amenta. Helly-type theorems and generalized linear programming. Discrete Comput. Geom., 12:241261, 1994.

[2] O. Cheong, X. Goaoc, and H.-S. Na. Geometric permutations of disjoint unit spheres. Comput. Geom. Theory Appl., 2005. In press.

[3] H. Debrunner. Helly type theorems derived from basic singular homology. Amer. Math. Monthly, 77:375-380, 1970.

[4] J. E. Goodman, R. Pollack, and R. Wenger. Geometric transversal theory. In J. Pach, editor, New Trends in Discrete and Computational Geometry, volume 10 of Algorithms and Combinatorics, pages 163198. Springer-Verlag, Heidelberg, Germany, 1993.

[5] B. Grünbaum. On common transversals. Arch. Math., 9:465-469, 1958.

[6] B. Grünbaum. Common transversals for families of sets. J. London Math. Soc., 35:408-416, 1960.

[7] H. Hadwiger. Wiskundige Opgaven, pages 27-29, 1957.

[8] H. Hadwiger. Über Eibereiche mit gemeinsamer Treffgeraden. Portugal Math., 6:23-29, 1957.

[9] E. Helly. Über Mengen konvexer Körper mit gemeinschaftlichen Punkten. Jahresbericht Deutsch. Math. Verein., 32:175-176, 1923.

[10] E. Helly. Über Systeme von abgeschlossenen Mengen mit gemeinschaftlichen Punkten. Monaths. Math. und Physik, 37:281-302, 1930.

[11] A. Holmsen, M. Katchalski, and T. Lewis. A Hellytype theorem for line transversals to disjoint unit balls. Discrete Comput. Geom., 29:595-602, 2003.

[12] A. Holmsen and J. Matoušek. No Helly theorem for stabbing translates by lines in $\mathbb{R}^{3}$. Discrete Comput. Geom., 31:405-410, 2004.

[13] M. Katchalski, S. Suri, and Y. Zhou. A constant bound for geometric permutations of disjoint unit balls. Discrete $\&$ Computational Geometry, 29:161-173, 2003.

[14] I. Macdonald, J. Pach, and T. Theobald. Common tangents to four unit balls in $\mathbb{R}^{3}$. Discrete Comput. Geom., 26:1-17, 2001.

[15] J. Matoušek. Using the Borsuk-Ulam Theorem. Springer-Verlag, 2003.

[16] H. Tverberg. Proof of Grünbaum's conjecture on common transversals for translates. Discrete Comput. Geom., 4:191-203, 1989.

[17] R. Wenger. Helly-type theorems and geometric transversals. In J. E. Goodman and J. O'Rourke, editors, Handbook of Discrete and Computational Geometry, chapter 4, pages 73-96. CRC Press LLC, Boca Raton, FL, 2nd edition, 2004. 